\title{
Fuzzy Evaluation Algorithm of Distribution Transformer Based on Adaptive Variable Weight
}

\author{
Yuling $\mathrm{Li}^{1}$, Erxia $\mathrm{Li}^{1}$, Yonghua Fan ${ }^{1}$, Chaoqun $\mathrm{Kang}^{1}$, Zheng Zhang ${ }^{2, *}$ and \\ Xinrui $\mathrm{HaO}^{2}$ \\ ${ }^{1}$ China Electric Power Science Research Institute, Beijing, China \\ ${ }^{2}$ School of Electrical Engineering, Wuhan University, China
}

\begin{abstract}
Considering status indicators degradation degree, a distribution transformer evaluation algorithm using adaptive variable weight is proposed based on fuzzy assessment. By analyzing the correlation of each single condition variable and the condition of transformer, condition variables are divided into important indexes and referential indexes. In terms of different condition variables, adaptive variable weight algorithm using suitable balance functions is built to calculate the weight coefficient separately. Combining existing fuzzy assessment procedures, an accurate transformer condition evaluation system is established using the variable weights obtained in this paper. The results of example showed that the proposed method can evaluate the condition of distribution transformer when referential index deviates from the normal state accurately.
\end{abstract}

Keywords: Adaptive variable weight; Fuzzy comprehensive evaluation; Important index; Referential index

\section{Introduction}

Distribution transformers are important equipment in the power distribution grid, and condition assessment plays an important role in safe operation of the power distribution grid. In practice, the staff needs to judge the condition of the transformer first, and then decide whether to repair the transformer. This process is called as condition assessment. In this process, the condition indexes of transformers will be collected and considered comprehensively in transformer condition assessment. Accurate condition assessment can save a lot of manpower and material resources, and can make the maintenance plan according to the actual condition of the equipment. Without accurate condition assessment, maintenance process will become blind and inefficient. Therefore, it has become a consensus that the maintenance based on condition assessment is very necessary.

In order to take the influences of all indexes into consideration, a suitable algorithm of information fusion is needed. The algorithms of information fusion for condition indexes include neural network method ${ }^{[1]}$, the gray clustering method ${ }^{[2]}$, the rough sets method ${ }^{[3]}$, Bayesian network method ${ }^{[4]}$, evidence reasoning method ${ }^{[5]}$, UV-Visible method ${ }^{[6]}$ and fuzzy logical method ${ }^{[7]}$. All above methods can satisfy the needs of transformer condition assessment, yet there are still some flaws in every method. The neural network method needs a number of data to train the neural network. Because accurate actual condition assessment records are difficult to obtain, so this method is not quite suitable for the actual operation. The modeling process of Bayesian network method is complex, and the probability is not easy to collect. Among them, fuzzy logical method in reference [7] is widely used because of its clear assessment process and good evaluation results.

In fuzzy logical method, the key step is to obtain the weight of indexes. Weight is the objective reflection of subjective measure on the relative importance of condition 
variables, so they are the important parameters of condition assessment system. However, some algorithms took the weight as a constant quantity, which is called constant weight method. Constant weight methods include the association rule ${ }^{[8]}$, the entropy-weighting method ${ }^{[9]}$ and analytic hierarchy process ${ }^{[10]}$. Among them, AHP is a simple and effective method to make analysis and comprehensive evaluation on complex problems that is multiple criteria, multi-factor and multi-level. In analytic hierarchy process, experts divide indexes into several layers according to the logic and compare two factors of each layer and get the judgment matrix.

Weight obtained by analytic hierarchy process will not change with the state of index, which is called constant weight. When certain indexes seriously deviated from their normal value, constant weight method couldn't reflect the transformer's operation condition accurately. Since constant weight methods have certain flaws, the variable weights get extensive attention of scholars. In order to adjust weights according to indexes' condition, variable weight method is proposed. This method utilizes the balance function of the relative degradation to change the constant weight. By this algorithm, the weight of some serious indexes can be increased, so that it can increase their influence on the assessment. Reference [11] proposed a typical punishment variable weight function of transformer condition assessment, yet this method couldn't reflect the difference among importance of indexes. A balance function was put forward by reference [8], but it couldn't distinguish the importance of indexes, especially when an index deviates from the normal value, that is to say, the degradation degree of important indexes of evaluation will be close to zero, the balance function value will tend to be infinity. The balance function in reference [8] can transform the weight well when important indexes degrade, but as unimportant index degrades, the corresponding weight coefficients value will be much larger than it should be. All of the above methods can't change the weight accurately. Without the classification based on importance of indexes, general change of weights will decrease the status of important indexes while increase the status of unimportant indexes. The result of this kind of assessment is not consistent with the actual situation.

According to the above analysis, this paper proposes a variable weight evaluation method based on classification of condition variables. Assessment indexes are categorized as important indexes and referential indexes based on the analysis of correlation between condition variables and transformer condition. Different variable weight balance functions are applied for different types of indexes. Based on existing fuzzy logic method, the transformer's operation condition can be evaluated according to the variable weight coefficient.

\section{Establishing of Evaluation Index System and Its Classification}

In order to assess distribution transformer's operation condition accurately, a distribution transformer evaluation index system needs establishing, which includes direct or indirect technical indicators, performance and operation parameters. Since distribution transformers are numerous, the evaluation indexes should be easy to collect. In practical, maintenance generally doesn't carry on dry-type transformer's condition assessment, so this paper focuses on the index system of oil-immersed transformer.

\subsection{Establishing Evaluation Index System}

Common faults of oil-immersed distribution transformer can be divided into winding fault, casing failure, and serious leakage of transformer oil, tap switch failure, fault caused by overvoltage, and core fault and so on. According to the "State Maintenance System Standard of Distribution Grid" issued by China State Grid Corp and the operation characteristics of distribution transformer, the condition evaluation system of distribution transformer is shown as Table 1. The evaluation system is divided into three layers: the 
target layer, the project layer and the index layer. The target layer is the operation condition of the distribution transformer, which can be recorded as A; the target layer includes eight project layers, which is denoted as: $U_{\mathrm{A} 1}-U_{\mathrm{A} 8}$; each project layer has several index layers, for example, the cooling $\operatorname{system}\left(\mathrm{A}_{3}\right)$ can be decomposed as two indexes, denoted as: $U_{\mathrm{A} 3}=\left(U_{\mathrm{A} 31}, U_{\mathrm{A} 32}\right)$.

Table 1. Evaluation Index System of Distribution Transformer

\begin{tabular}{|c|c|c|}
\hline Target layer & Project layer & Index layer \\
\hline \multirow{23}{*}{$\begin{array}{c}\text { Running } \\
\text { Condition } \\
\text { of } \\
\text { Transformer }\end{array}$} & \multirow{8}{*}{$\begin{array}{l}\text { Winding } \\
\text { and } \\
\text { Casing } \\
\mathrm{A}_{1}\end{array}$} & DC resistance $A_{11}$ \\
\hline & & Insulation resistance $\mathrm{A}_{12}$ \\
\hline & & Joint temperature $\mathrm{A}_{13}$ \\
\hline & & Load ratio $\mathrm{A}_{14}$ \\
\hline & & Filth $\mathrm{A}_{15}$ \\
\hline & & Appearance Complete $\mathrm{A}_{16}$ \\
\hline & & Body temperature $\mathrm{A}_{17}$ \\
\hline & & Unbalanced current $\mathrm{A}_{18}$ \\
\hline & Tap Switch $\mathrm{A}_{2}$ & Tap switch $\mathrm{A}_{21}$ \\
\hline & \multirow[t]{2}{*}{$\begin{array}{c}\text { Cooling System } \\
\mathrm{A}_{3}\end{array}$} & $\begin{array}{l}\text { Temperature control operation } \\
\mathrm{A}_{31}\end{array}$ \\
\hline & & Fan operation $\mathrm{A}_{32}$ \\
\hline & \multirow{5}{*}{$\begin{array}{l}\text { Oil Tank } \\
\mathrm{A}_{4}\end{array}$} & Distance to ground $\mathrm{A}_{41}$ \\
\hline & & Seal up $\mathrm{A}_{42}$ \\
\hline & & Oil level $\mathrm{A}_{43}$ \\
\hline & & Oil temperature $\mathrm{A}_{44}$ \\
\hline & & Rust $\mathrm{A}_{45}$ \\
\hline & Non-electricity Protection $\mathrm{A}_{5}$ & Non-electricity protection $\mathrm{A}_{51}$ \\
\hline & \multirow{2}{*}{$\begin{array}{c}\text { Grounding } \\
\mathrm{A}_{6} \\
\end{array}$} & Down-conductor protection $\mathrm{A}_{61}$ \\
\hline & & Grounding resistance $\mathrm{A}_{62}$ \\
\hline & \multirow{2}{*}{$\begin{array}{l}\text { Insulating Oil } \\
\qquad \mathrm{A}_{7}\end{array}$} & Insulating oil color $\mathrm{A}_{71}$ \\
\hline & & Withstand voltage test $\mathrm{A}_{72}$ \\
\hline & \multirow{2}{*}{$\begin{array}{l}\text { Maintenance Record and } \\
\text { Identification } A_{8}\end{array}$} & Identification $\mathrm{A}_{81}$ \\
\hline & & Similar maintenance history $\mathrm{A}_{82}$ \\
\hline
\end{tabular}

\subsection{Important Index and Referential Index}

According to the relations between the evaluation indexes and the operation condition, the evaluation indexes can be divided into important indexes and referential indexes.

In terms of important indexes, they can directly reflect the condition of transformers. Once these indexes are seriously deteriorated, operation condition of the transformer can be ensured that the transformer is in fault. Taking the cooling system of transformer for example, if it is seriously deteriorated, the transformer is in fault condition according to the experience, and needs emergence repairing. In terms of referential indexes, they can reflect transformer's operation condition indirectly. When these indexes are seriously deteriorated, the transformer may be in a fault condition, but it cannot directly indicate the fault condition of transformer. When the "family defects" of equipment such as transformer maintenance are abnormal, it suggests that this kind of transformer may have designing flaws. However, the conclusion that the transformer is in fault condition cannot be drawn only based on referential index of serious deterioration. The reason is that different working conditions cause the difference of maintenance among transformer.

Based on the correlation between the evaluation process of distribution transformers and indexes, indexes of the project layer $\mathrm{A}_{1}-\mathrm{A}_{7}$ and their subordinate indexes are regarded 
as important indicators, while project layer index $\mathrm{A}_{8}$ and their subordinate indexes are referential indexes.

\section{Standardization of Evaluation Index $X$}

The transformer condition evaluation indexes can also be divided into quantitative indexes and qualitative indexes according to whether the data can be recorded. Quantitative indexes can be recorded by data, but it is difficult to be compared directly because of the difference in their dimension and order of magnitude, while qualitative indexes are difficult to be recorded by data. In order to compare the index with each other and provide the calculation basis for the subsequent variable weight process, the concept of the deterioration degree is introduced to reflect the operation condition of indexes [12]. The deterioration degree $x_{i}$ is defined from 0 to 1 , the degree of deterioration is better if it is closer to 1 .

\subsection{Quantitative Index}

In terms of quantitative indexes, they can be divided into 2 types with different properties.

One type of indexes' measured value is higher if the indexes are in better condition, and their deterioration degree can be obtained in accordance with formula (1).

$$
x_{i}=\frac{V_{i}-V_{i \min }}{V_{i \max }-V_{i \min }}
$$

Another type of indexes' measured value is lower if the indexes are in better condition, so their deterioration degree can be obtained in accordance with formula (2).

$$
x_{i}=\frac{V_{i \max }-V_{i}}{V_{i \max }-V_{i \min }}
$$

In formula (1) and (2), $x_{i}$ is the degradation degree of index $i ; V_{i \max }$ is maximum value of index $i$ while $V_{i \min }$ is the minimum value, which can be referred in "test rule on the prevention of electrical equipment" and "the power transformer operation rules ", $V_{i}$ is the measured value of the $i$-th index.

\subsection{Qualitative Index}

Indexes that can't be described by data directly are called qualitative indexes, such as operating data, maintenance records. Qualitative indexes' degree of deterioration is obtained by expert scoring method. Based on their experience, experts give every qualitative index a score from 0 to 1 , and the index will be better if the score is closer to 1 . According to experts' level of technology and experience, the corresponding weights $\rho$ are given to experts respectively. Qualitative indexes' degree of deterioration can be obtained in accordance with the formula (3).

$$
x_{i}=\sum_{j=1}^{m} x_{i j} \rho_{j}
$$

Where, $x_{i}$ is the degree of deterioration for $i$-th qualitative index, $x_{i j}$ is the $j$-th expert's score on the $i$-th index, $m$ is the number of experts, $\rho_{j}$ is the $j$-th expert's weight value, which is bigger if the expert has more prestige and experience, and the values meet $\sum_{i=1}^{m} \rho_{i}=1$.

\section{Calculation of Variable Weight Coefficient}

In fuzzy evaluation method, obtaining suitable weight coefficients is the key of accurate evaluation. In order to reflect the relation between indexes and operation condition of transformer better, this paper proposes the adaptive balance function based 
on classified indexes to calculate the variable weight coefficient. First, constant weight coefficients of index layer and project layer can be obtained by analytic hierarchy process. Then, the variable weights is calculated according to the adaptive balance function.

\subsection{Basic Principle of Variable Weight}

The actual operation condition of the distribution transformer has the "buckets effect", that is, how much water a wooden bucket can hold doesn't depend on the average length of all boards, but depends on the shortest piece of wood. When the transformer's important index is in serious deterioration condition, its operation condition is likely to reach a serious condition. Therefore, in order to reflect the distribution transformer's "buckets effect ", Chinese Professors proposed the variable weight theory [13]. Then, in 1995 based on the theory of spatial factors, the theory of variable weight was proposed by Professor Li Hongxing, who analyzed three kinds of condition variable weight vector of the axiomatic definition. The condition variable weight vectors can be divided into various types, and the punishment type variable weight vector is more suitable for the condition evaluation of distribution transformer. Because punishment type variable weight vectors change relatively slowly on higher value index but have sensitive reaction to the lower value evaluation index, so it can describe the transformer's "buckets effect" accurately.

Constant weight coefficient is transferred to the variable weight coefficient based on Hadamard product according to the formula (4).

$$
w_{i}=\frac{w_{i 0} f\left(x_{i}\right)}{\sum_{p=1}^{n} w_{p 0} f\left(x_{p}\right)}
$$

Where, $f\left(x_{i}\right)$ is the balance function with the $i$-th index's degree of deterioration, reflecting how much the corresponding constant weight is magnified, $w_{i 0}$ is the constant weight coefficient of the $i$-th index, and $w_{i}$ is the variable weight coefficient after normalized.

\subsection{Selection of Variable Weight Balance Function}

This paper adopts formula (5) to judge the weight-changing ability of balance function by adjusting degree $D(X)[14]$.

$$
D(X)=\frac{1}{2} \sum_{j=1}^{m}\left(w_{j}-w_{j 0}\right)^{2}
$$

Where, $X$ is the weight vector with $m$ weight coefficients, $w_{j 0}$ is the constant weight coefficient of the $j$-th index, and $w_{j}$ is the variable weight coefficient obtained by the balance function.

In the evaluation practice, if the index system needs to be more balanced, then the adjusting degree of the balance function should be high. Otherwise, the state variable weight vector with a slightly lower adjusting degree should be selected. Thus, the adjusting degree of important indexes' balance function should be larger than that of the referential indexes. The physical meaning of variable weights is that when an important index deviates from the normal value, the weight coefficient will be enlarged to reflect its influence on the real condition of the transformer. If a referential index is seriously deviated from the normal value, the weight of the index should be appropriately enlarged to reflect its proper influence on the condition of the transformer, but the importance of the referential index shouldn't be over-exaggerated in the process.

\subsection{Comparison of Variable Weight Balance Function}

According to the above analysis, the balance function $f(x)$ of the distribution transformer selected should have the following properties:

1) Continuous and differentiable; 
2) Can be well fitted with the real assessment result;

3) Simple form;

4) The adjusting degree of $f(x)$ corresponding to important index is relatively large;

5) The adjusting degree of $f(x)$ corresponding to referential index is relatively small.

Based on these properties, this paper selects four typical punishing functions as the balance function according to important indexes and reference indexes, including power function, linear, parabolic and exponential, whose formulas are shown as: (6)-(9)

$$
\begin{gathered}
f(x)=x^{-0.5} \\
f(x)=3-2 x \\
f(x)=3(x-1)^{2}+1 \\
f(x)=\frac{1}{1-e^{-x}}
\end{gathered}
$$

Balance functions can be compared according to their adjusting degree. The calculation of the adjusting degree depends on the deterioration degree and indexes' constant weight. In this paper, the variable weight method is to highlight the "buckets effect" of transformer. So we made a reasonable assumption in this paper. Assuming that there are 8 indexes in total, and the important degree of each index is the same, of which constant weight is 0.125 . In the evaluation, an index is severely degraded, and its degradation degree is 0.1 , other 7 indexes are 0.8 . The adjusting degree of different balance functions are calculated and shown in Table 2.

According to Table 2, it can be seen that the minimum and the maximum adjusting degree belongs to balance function in formula (7) and formula (9). The evaluation result of the important indicators demands high balance, yet the balance of the referential index is of low demand. Therefore, the adjusting degree of the balance function of the important index should be high yet the referential index is low.

\section{Table 2. Variable Weight and Adjusting Degree of Different Balance Functions}

\begin{tabular}{ccccc}
\hline & Function(6) & Function (7) & Function(8) & Function(9) \\
\hline Index1(degraded) & 0.2878 & 0.2222 & 0.3043 & 0.4526 \\
Index 2 & 0.1017 & 0.1111 & 0.0994 & 0.0782 \\
Index 3 & 0.1017 & 0.1111 & 0.0994 & 0.0782 \\
Index 4 & 0.1017 & 0.1111 & 0.0994 & 0.0782 \\
Index 5 & 0.1017 & 0.1111 & 0.0994 & 0.0782 \\
Index 6 & 0.1017 & 0.1111 & 0.0994 & 0.0782 \\
Index 7 & 0.1017 & 0.1111 & 0.0994 & 0.0782 \\
Index 8 & 0.1017 & 0.1111 & 0.0994 & 0.0782 \\
Adjusting degree & 0.0152 & 0.0054 & 0.0184 & 0.0613 \\
\hline
\end{tabular}

Based on the above analysis, the adaptive variable weight theory can reflect the balance of indexes in comprehensive evaluation more effectively. Therefore, the function in $(10 a)$ is selected as variable weight formula for important indexes.

$$
w_{i}=\frac{w_{i 0}}{1-e^{-x_{i}}} / \sum_{p=1}^{n} \frac{w_{p 0}}{1-e^{-x_{p}}}
$$

Where, $w_{i 0}$ is the constant weight coefficient of the $i$-th index, $w_{i}$ is variable weight coefficient, $x_{i}$ is the deterioration degree of the $i$-th index and $n$ is the number of indexes.

Referential indexes' variable weight formula is shown as $(10 b)$.

$$
w_{i}=\frac{w_{i 0}\left(b-a x_{i}\right)}{\sum_{p=1}^{n} w_{p 0}\left(b-a x_{p}\right)}
$$


Where, $a$ and $b$ are in accordance with the correlation of the referential indexes and operation condition, $b$ is higher if the referential index has more relation with transformer condition, $a$ reflects the decay rate of referential index's weight.

Adaptive variable weight method has the advantage that if the important index is of serious deterioration and its deterioration degree is rather lower than other indexes'. According to formula (10a) for automatically adjusting weight coefficient, the variable weight coefficient is bigger when the index has lower deterioration degree. When the referential indexes are of serious deterioration, according to formula $(10 b)$, the weight coefficient of index can be adjusted automatically and its amplification ratio is limited without excessive amplification. Thus, this method can reflect the role of referential index in condition assessment process accurately.

\section{Choice of Membership Function}

In fuzzy logical method, a fuzzy evaluation set $V=\left\{v_{1}, v_{2}, v_{3}, v_{4}\right\}$ should be established, elements in $V$ are representatives of the operation condition of transformer: good, general, attention and serious. The membership function in fuzzy theory is used to determine the evaluation factors $\left\{U_{1}, U_{2}, \ldots U_{n}\right\}$. For the quantitative index, the membership degree is determined by the distribution function of the triangle and the semi-trapezoidal combination as shown in Figure 1. Taking the insulation resistance of the windings as an example, the membership functions of the corresponding four conditions are obtained, as is shown in functions (11)-(14). Methods of other quantitative indexes to construct membership function are the same as above functions.

$$
\begin{aligned}
& \mu(x)=\left\{\begin{array}{l}
1, x \geq 0.8 \\
5 x-3,0.6<x<0.8 \\
0, x \leq 0.6
\end{array}\right. \\
& \mu(x)=\left\{\begin{array}{l}
4-5 x, 0.6 \leq x<0.8 \\
5 x-2,0.4<x<0.6 \\
0, x \leq 0.4 \text { or } x \geq 0.8
\end{array}\right. \\
& \mu(x)=\left\{\begin{array}{l}
3-5 x, 0.4 \leq x<0.6 \\
5 x-1,0.2<x<0.4 \\
0, x \leq 0.2 \text { or } x \geq 0.6
\end{array}\right. \\
& \mu(x)=\left\{\begin{array}{l}
2-5 x, 0.2<x<0.4 \\
1, x \leq 0.2 \\
0, x \geq 0.4
\end{array}\right.
\end{aligned}
$$

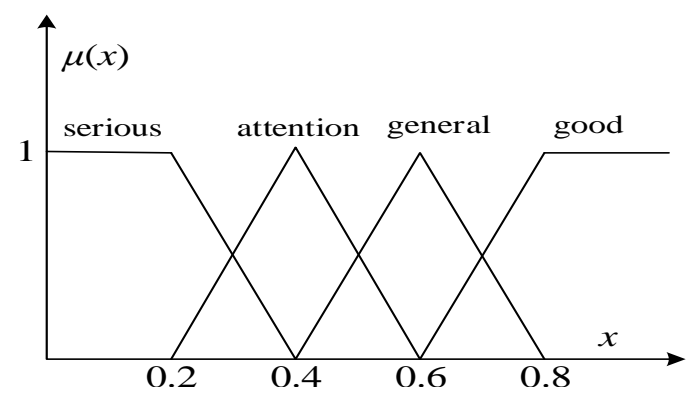

Figure 1. Membership Functions of a Certain Quantitative Index

For qualitative indexes, the membership degree can be obtained by expert scoring method according to formula (15). 


$$
r_{i j}=\frac{m_{i j}}{n}
$$

Where, $r_{i j}$ is the membership degree of the $i$-th index for the $j$-th state, $m_{i j}$ is the number of experts who agree the $i$-th index belongs to the $j$-th state, and $n$ is the number of indexes.

\section{Procedures of Transformer Assessment}

The process of adaptive variable weight transformer evaluation based on index classification is summarized as follows:

Step 1 Input transformer single state information in Table 1.

Step 2 Calculate $x_{i}$ according to deterioration degree of each index in the index layer.

Step 3 Calculate judgment matrix $G$ and constant weight of each index layer $w_{i 0}$ based on analytic hierarchy process.

Step 4 Choose the adaptive balance function according to different types of indexes.

Step 5 According to formula (4), the variable weights of the index layer $w_{i}$ and the weight vector are obtained.

Step 6 Establish the transformer operation condition including good, general, attention and serious, then calculate the membership degree of the single index to four state levels and obtain membership matrix $R_{i}$.

Step 7 Calculate the evaluation vector of project level using $B_{i}=W_{i} \cdot R_{i}$.

Step 8 Regard mean value of the comprehensive evaluation vector of the project layer as its deterioration degree and then obtain variable weight coefficient of project layer $W$ according to the formula (4). If $B_{i}=(a, b, c, d)$, the mean value of $B_{i}$ can be obtained according to formula (16) .

$$
E\left(B_{i}\right)=0.875 a+0.625 b+0.375 c+0.125 d
$$

Step 9 Formula (17) is used to calculate the state evaluation vector $B$ to obtain the overall state of the transformer. In formula (17), $W$ represents for project layer weight coefficient. The project layer membership degree matrix $R$ is obtained according to formula (18).

$$
\begin{gathered}
B=W \cdot R \\
R=\left[B_{1}, B_{2}, B_{3}, B_{4}, B_{5}, B_{6}, B_{7}, B_{8}\right]^{T}
\end{gathered}
$$




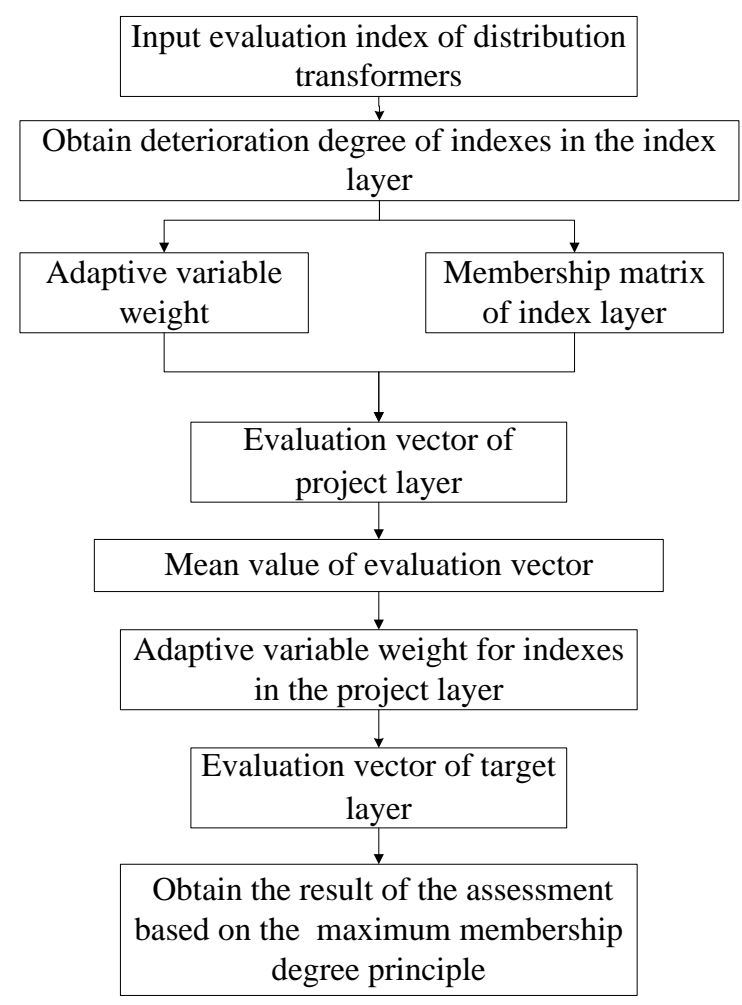

Figure 2. Evaluation Process of Distribution Transformer

\section{Example Analysis}

In order to verify the validity of the adaptive variable weight condition evaluation method of distribution transformer, a $10 \mathrm{kV}$ distribution transformer was selected as an example.

\subsection{Pre-processing of Data}

Input the data of index layer in Table 1, the normalized deterioration degree of distribution transformer index can be obtained according to the formula (1)-(3), as is shown in Table 3. It is not difficult to see that two referential indexes $A_{81}, A_{82}$ are seriously deteriorated. The degradation degree is used to calculate the variable weight coefficients in the next step.

The constant weight of the index layer should be obtained based on the existing methods. A lot of methods can be used to obtain the constant weight, and we used the analytic hierarchy process to obtain it in this paper. To avoid repetition, it' not described in this article.

We transformed the constant weight of the index layer based on formula (10). In order to better illustrate the problem, we toke the literature [8] as an example, the results of this paper are compared with the results of literature [8].

As is mentioned, we obtained the constant weight of indexes by analytic hierarchy process. Based on the classification of indexes, the weight can be changed according to the formula (10). In this process, the degradation degree of each index is the input of the balance function. In order to avoid repetition, we will show the specific process of variable weight in the subsequent process. Here, we listed constant weights and variable weights in Table 4. 
Table 3. Deteriorations of Index Layer

\begin{tabular}{cc}
\hline Index & Degree of deterioration \\
\hline DC resistance $\mathrm{A}_{11}$ & 0.6013 \\
Insulation resistance $\mathrm{A}_{12}$ & 0.5700 \\
Joint temperature $\mathrm{A}_{13}$ & 0.4960 \\
Load ratio $\mathrm{A}_{14}$ & 0.4833 \\
Filth $\mathrm{A}_{15}$ & 0.4531 \\
Appearance Complete $\mathrm{A}_{16}$ & 0.5524 \\
Body temperature $\mathrm{A}_{17}$ & 0.5694 \\
Unbalanced current $\mathrm{A}_{18}$ & 0.5689 \\
Tap switch $\mathrm{A}_{21}$ & 0.5725 \\
Temperature control operation $\mathrm{A}_{31}$ & 0.5623 \\
Fan operation $\mathrm{A}_{32}$ & 0.4634 \\
Distance to ground $\mathrm{A}_{41}$ & 0.5253 \\
Seal up $\mathrm{A}_{42}$ & 0.5754 \\
Oil level $\mathrm{A}_{43}$ & 0.5379 \\
Oil temperature $\mathrm{A}_{44}$ & 0.5240 \\
Rust $\mathrm{A}_{45}$ & 0.6023 \\
Non-electricity protection $\mathrm{A}_{51}$ & 0.5834 \\
Down-conductor protection $\mathrm{A}_{61}$ & 0.5345 \\
Grounding resistance $\mathrm{A}_{62}$ & 0.5973 \\
Insulating oil color $\mathrm{A}_{71}$ & 0.5309 \\
Withstand voltage test $\mathrm{A}_{72}$ & 0.4992 \\
Identification $\mathrm{A}_{81}$ & 0.1250 \\
Similar maintenance history $\mathrm{A}_{82}$ & 0.1000 \\
\hline
\end{tabular}

Table 4. Variable Weights According to Different Balance Functions

\begin{tabular}{cccc}
\hline Index & $\begin{array}{c}\text { Constant } \\
\text { weight }\end{array}$ & $\begin{array}{c}\text { Traditional } \\
\text { method [8] }\end{array}$ & Proposed method \\
\hline DC resistance $\mathrm{A}_{11}$ & 0.1920 & 0.1742 & 0.1141 \\
Insulation resistance $\mathrm{A}_{12}$ & 0.1680 & 0.1608 & 0.1187 \\
Joint temperature $\mathrm{A}_{13}$ & 0.1090 & 0.1199 & 0.1319 \\
Load ratio $\mathrm{A}_{14}$ & 0.0880 & 0.0993 & 0.1346 \\
Filth $\mathrm{A}_{15}$ & 0.0730 & 0.0879 & 0.1415 \\
Appearance Complete $\mathrm{A}_{16}$ & 0.1060 & 0.1047 & 0.1215 \\
Body temperature $\mathrm{A}_{17}$ & 0.1050 & 0.1006 & 0.1188 \\
Unbalanced current $\mathrm{A}_{18}$ & 0.1590 & 0.1525 & 0.1189 \\
Tap switch $\mathrm{A}_{21}$ & 1.0000 & 1.0000 & 1.0000 \\
Temperature control & 0.7500 & 0.7120 & 0.7212 \\
operation $\mathrm{A}_{31}$ & 0.2500 & 0.2880 & 0.2788 \\
Fan operation $\mathrm{A}_{32}$ & 0.1626 & 0.1697 & 0.1679 \\
Distance to ground $\mathrm{A}_{41}$ & 0.2257 & 0.2151 & 0.2177 \\
Seal up $\mathrm{A}_{42}$ & 0.2535 & 0.2584 & 0.2571 \\
Oil level $\mathrm{A}_{43}$ & 0.2257 & 0.2362 & 0.2335 \\
Oil temperature $\mathrm{A}_{44}$ & 0.1326 & 0.1207 & 0.1237 \\
Rust $\mathrm{A}_{45}$ & 1.0000 & 1.0000 & 1.0000 \\
Non-electricity protection & & & \\
$\mathrm{A}_{51}$ & 0.3670 & 0.3932 & 0.3864 \\
Down-conductor & 0.6330 & 0.6068 & \\
protection $\mathrm{A}_{61}$ & & & 0.6136 \\
\hline Grounding resistance $\mathrm{A}_{62}$ & & & \\
\hline & & & \\
\hline
\end{tabular}




\begin{tabular}{cccc}
\hline Insulating oil color $\mathrm{A}_{71}$ & 0.2540 & 0.2425 & 0.2452 \\
Withstand voltage test $\mathrm{A}_{72}$ & 0.7460 & 0.7575 & 0.7548 \\
Identification $\mathrm{A}_{81}$ & 0.5000 & 0.4444 & 0.4955 \\
Similar maintenance & 0.5000 & 0.5556 & 0.5045 \\
history $\mathrm{A}_{82}$ & & & \\
\hline
\end{tabular}

\subsection{Preparation for Variable Weight of Project Layer}

We have just completed the variable weight of the index layers. In order to get the final evaluation results, it is necessary to obtain the variable weight of the project layer. In order to achieve this goal, we need to get the evaluation vector of the index layer, and then calculate the mean value of each index layer, so as to change the weight of the project layer.

First, we calculate the evaluation vectors of the project layers. According to the membership function, the membership matrix of each index layer $R_{i}$ is obtained, and then get the evaluation vector of project layer $B_{i}$. Based on the traditional balance function and the balance function in this paper, the evaluation vector $B_{i}$ of the project layer can be calculated, and the corresponding results are shown in Table 5.

Table 5. Evaluation Vectors of Project Layers According to Different Balance Functions

\begin{tabular}{|c|c|c|}
\hline Project Layer & Traditional method $^{[8]}$ & Proposed method \\
\hline Winding and Casing $A_{1}$ & $(0.0116,0.2868,0.5494,0.1522)$ & $(0.0129,0.2503,0.5759,0.1609)$ \\
\hline Tap Switch $\mathrm{A}_{2}$ & $(0,0.3500,0.6500,0)$ & $(0,0.3500,0.6500,0)$ \\
\hline Cooling System $\mathrm{A}_{3}$ & $(0.0712,0.1856,0.5000,0.2432)$ & $(0.0721,0.1861,0.5000,0.2418)$ \\
\hline Oil Tank $\mathrm{A}_{4}$ & $(0.0121,0.2018,0.5599,0.2263)$ & $(0.0124,0.20220 .5598,0.2255)$ \\
\hline Non-electricity Protection $\mathrm{A}_{5}$ & $(0.1000,0.1000,0.6000,0.2000)$ & $(0.1000,0.1000,0.6000,0.2000)$ \\
\hline Grounding $\mathrm{A}_{6}$ & $(0.0303,0.2000,0.5714,0.1983)$ & $(0.0307,0.2000,0.5727,0.1966)$ \\
\hline Insulating $\mathrm{Oil}_{7}$ & $(0.1243,0.2000,0.4758,0.2000)$ & $\begin{array}{c}(0.1245,0.2000 \\
0.4755,0.2000)\end{array}$ \\
\hline $\begin{array}{c}\text { Maintenance Record and } \\
\text { Identification } A_{8}\end{array}$ & $(0,0,0,1)$ & $(0,0,0,1)$ \\
\hline
\end{tabular}

Secondly, the mean value of each project layer can be obtained according to the evaluation vector of the project layer. The mean values of the project layer's evaluation vector are obtained according to formula (18) and the results are shown in Table 6.

Table 6. Mean Values of Evaluation Vectors According to Different Balance Functions

\begin{tabular}{|c|c|c|}
\hline Project Layer & Traditional method & Proposed method \\
\hline Winding and Casing $\mathrm{A}_{1}$ & 0.4145 & 0.4038 \\
\hline Tap Switch $\mathrm{A}_{2}$ & 0.4625 & 0.4625 \\
\hline Cooling System $\mathrm{A}_{3}$ & 0.3962 & 0.3971 \\
\hline Oil Tank $\mathrm{A}_{4}$ & 0.3750 & 0.3753 \\
\hline Non-electricity Protection $\mathrm{A}_{5}$ & 0.4000 & 0.4000 \\
\hline Grounding $\mathrm{A}_{6}$ & 0.3906 & 0.3912 \\
\hline Insulating Oil $\mathrm{A}_{7}$ & 0.4372 & 0.4373 \\
\hline Maintenance Record and Identification $\mathrm{A}_{8}$ & 0.1250 & 0.1250 \\
\hline
\end{tabular}


Next, by analytic hierarchy process, the weight coefficients of project layer in the distribution transformer are obtained as $0.2,0.1,0.1,0.2,0.1,0.1,0.1,0.1$ respectively.

Finally, we can change the weight of the project layer.

\subsection{Variable Weight of Project Layer}

The mean value of project evaluation vector is regarded as its deterioration degree. The variable weight coefficients of different balance functions of project layers can be calculated by formula (4). Variable weight coefficients obtained by traditional balance function are $0.1298,0.0616,0.0736,0.1666,0.0828,0.0848,0.0758,0.3550$ respectively, while variable weight coefficients obtained by the ones presented in this paper are 0.2033 , $0.0912,0.1030,0.2159,0.1024,0.1043,0.0953$ and 0.0844 , as shown in Figure 3.

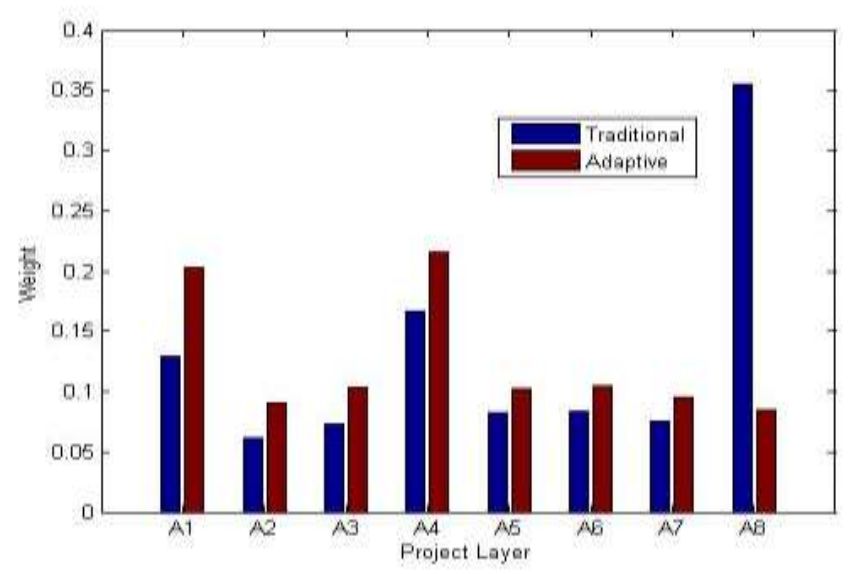

Figure 3. Variable Weights According to Different Balance Functions

As is shown in Figure 3, for important indexes $A_{1}-A_{7}$, variable weight coefficients differ a little in accordance with the traditional balance function and the ones proposed in this paper, which suggests that adaptive variable weight method can deal with assessment of important indexes well. For reference index $\mathrm{A}_{8}$, the variable weight coefficients obtained by traditional balance function and the ones presented in this paper differ a lot. Among them, the numerical value of the coefficient has been seriously deviated from the weight coefficient by traditional variable weight method. However, the weight obtained by the method of variable weight proposed in this paper is suitably enlarged on the basis of constant weight.

\subsection{Analysis of the Assessment Result}

We can get the target layer condition assessment vector $B$ toward different variable weight methods according to formula (16), respectively, as is shown in Figure 4.

$$
\begin{gathered}
B \text { Traditional }=(0.0290,0.1465,0.3756,0.4789) \\
B \text { Adaptive }=(0.0380,0.1958,0.5152,0.2507)
\end{gathered}
$$

In Figure 4, we can clearly see that the adaptive algorithm and the traditional variable weights differ in the transformer condition. The adaptive algorithm proposed in this paper concluded that the transformer was in the attention state, which was consistent with the fact.

According to the transformer condition evaluation vector obtained by traditional variable weight method, it can be seen that the fourth grade's membership is the largest, and we can conclude that the distribution transformer is in the fault condition based on the maximum membership degree principle of evaluation, but the result isn't in conformity with the actual operation condition. According to the method proposed in this paper, it 
can be seen that the membership of the third grade is far outweigh the other three grades, so the maintenance personnel can safely judge the distribution transformer is in attention condition based on the principle of maximum membership degree. The algorithm proposed by this paper not only embodies the indexes' influence to the weight but also the difference of indexes' importance. Therefore, the adaptive algorithm is still able to obtain accurate evaluation results in some serious deterioration of the index, which verifies the superiority and effectiveness of the method.

Therefore, in the case of seriously deteriorated referential indexes, the maintenance personnel can judge transformer operation state based on the variable weight method proposed in this paper quickly and accurately, and determine further maintenance plan, which reflects the advantage of the adaptive variable weighting method.

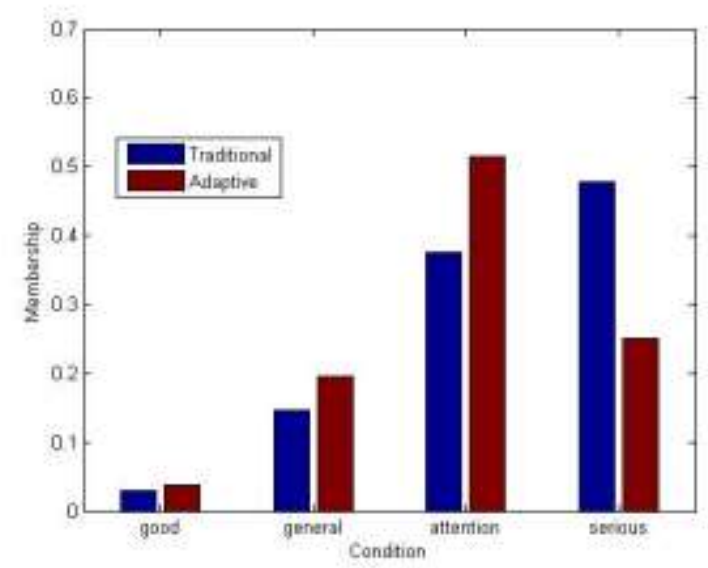

Figure 4. Evaluation Vectors According to Different Balance Functions

\section{Conclusion}

In distribution transformer condition assessment, adaptive variable weighting algorithm for distribution transformer condition assessment is used in this paper, evaluation results are accurate and reasonable, and the main conclusions are as follows:

1) According to the example analysis, we verified that in the condition assessment of the transformer, it is necessary to change the weight of each index of the transformer to make the result more accurate. What's more, classification of the indexes according their importance is consistent with the actual situation. Balance functions should be chosen in terms of different kinds of indexes.

2) By calculating, we proved that we should select the balance function which has bigger adjusting degree for important indexes. On the contrary, we should select the balance function which has smaller adjusting degree for the referential indexes.

3) A classification method for distribution transformer evaluation index is proposed, and the indexes can be divided into the important index and the referential index, which provide the basis for the adaptive variable weight.

4) Based on the variable weight fuzzy evaluation method and adaptive balance function, the variable weights are obtained. If referential indexes of distribution transformer are of serious deterioration, it is still able to evaluate the state effectively by the method proposed in this paper.

5) Although this paper is based on the research of the transformer, the adaptive variable weight algorithm proposed in the paper also has important reference value for the condition assessment of other kind of power distribution equipment. 


\section{References}

[1] A. E. B. Abu-Elanien, M. M. A. Salama and M. Ibrahim, "Determination of Transformer Health Condition Using Artificial Neural Networks", 2011 International Symposium on Innovations in Intelligent Systems and Applications (INISTA), Istanbul, Turkey, (2011).

[2] W. Zhao and Y. Zhu, "A Prediction Model for Dissolved Gas in Transformer Oil Based on Improved Verhulst Grey Theory”, 2008 3rd IEEE Conference on Industrial Electronics and Applications, Singapore, Singapore, (2008).

[3] Z. Ming and W. Taiyong, "Fault Diagnosis of Power Transformer Based on Association Rules Gained by Rough Set", 2010 The 2nd International Conference on Computer and Automation Engineering (ICCAE), Singapore, Singapore, (2010).

[4] G. Zheng and Z. Yongli, "Research Of Transformer Fault Diagnosis Based On Bayesian Network Classifiers", 2010 International Conference on Computer Design and Applications (ICCDA), Qinhuangdao, China, (2010).

[5] W. H. Tang, K. Spurgeon, Q. H. Wu and Z. J. Richardson, "An Evidential Reasoning Approach to Transformer Condition Assessments", IEEE Transactions on Power Delivery, vol. 19, no. 4, (2004), pp. 1696-1703.

[6] K. Hussain and Subrata Karmakar, "Condition assessment of transformer oil using UV-Visible spectroscopy”, 2014 Eighteenth National Power Systems Conference (NPSC), Guwahati, India, (2014).

[7] B. Németh, S. Laboncz and I. Kiss, "Condition Monitoring of Power Transformers using DGA and Fuzzy Logic”, 2009 IEEE Electrical Insulation Conference, Montreal, Canada, (2009).

[8] L. Li, X. Longjun, Z. Deng, Y. Bin, G. Yafeng and L. Fuchang, "Condition Assessment of Power Transformers Using a Synthetic Analysis Method Based on Association Rule and Variable Weight Coefficients", IEEE Transactions on Dielectrics and Electrical Insulation, vol.20, no.6, (2013), pp. 2052-2060.

[9] H. Lu, Y. Ye and G. Chen, "Transformer Fault Diagnosis Algorithm Based on Entropy-Weighting Information Bottleneck Method", 2015 International Conference on Cyber-Enabled Distributed Computing and Knowledge Discovery (CyberC), Xi' an, China, (2015).

[10] M. Bartłomiejczyk, M. Gutten and S. Hamacek, "Analysis of transformer state by fuzzy TOPSIS and AHP method", Proceedings of the 2014 15th International Scientific Conference on Electric Power Engineering (EPE), Brno, Czech, (2014).

[11] R. Liao, H. Zheng, S. Grzybowski, L. Yang, Y. Zhang, and Y. Liao, “An Integrated Decision-Making Model for Condition Assessment of Power Transformers Using Fuzzy Approach and Evidential Reasoning”, IEEE Transactions on Power Delivery, vol. 26, no. 2, (2011), pp. 1111-1118.

[12] H. Ma, L. Zhou, L. Liu and Y. Bao, "Notice of Retraction Research on equipment health state evaluation model based on deterioration degree", 2013 International Conference on Quality, Reliability, Risk, Maintenance, and Safety Engineering (QR2MSE), Chengdu, China, (2013).

[13] Z. Xu and Y. Zhuang, "The construction and application of variable weight vector", 7th World Congress on Intelligent Control and Automation, Chongqing, China, (2008).

[14] L. De-qing and H. Fei-long, "Weights Transferring Effect of State Variable Weight Vector", Systems Engineering - Theory \& Practice Online, vol. 29, no.6, (2009), pp. 127-131.

\section{Authors}

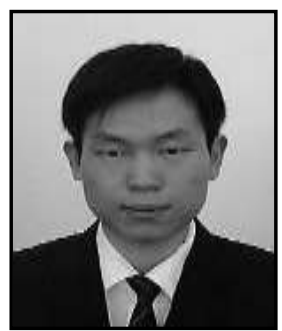

Yuling Li (1985-), is a researcher in the power distribution department of China Electric Power Research Institute. His major field of study is power distribution system condition-based maintenance and power distribution automation. E-mail: liyuling@epri.sgcc.com.cn

Erxia $\mathbf{L i}$ (1984-), is a researcher in the power distribution department of China Electric Power Research Institute. Her major field of study is power distribution system condition-based maintenance and power distribution automation. E-mail: lierxia@epri.sgcc.com.cn

Yonghua FAN(1974-), is a researcher in the power distribution department of China Electric Power Research Institute. His major field of study is power distribution system condition-based maintenance and power distribution automation. E-mail: 
fanyonghua@epri.sgcc.com.cn

Chaoqun Kang:(1991-), is a researcher in the power distribution department of China Electric Power Research Institute. Her major field of study is power distribution system condition-based maintenance. E-mail: kangchaoqun@epri.sgcc.com.cn

Zheng Zhang(1993-), is a master in the school of electrical engineering of Wuhan University. His major field of study is power distribution system condition-based maintenance and power distribution automation. E-mail: whu_zhangzheng@163.com

Xinrui Hao(1991-), is a master in the school of electrical engineering of Wuhan University. His major field of study is power distribution system condition-based maintenance and power distribution automation. E-mail: haoxinrui2009@163.com. 
International Journal of Control and Automation Vol.10, No.8 (2017) 